Revista Brasileira de Meteorologia, v.26, n.2, 273 - 278, 2011

\title{
COMPARAÇÃO DA TEMPERATURA DO AR OBTIDA POR ESTAÇÃO METEOROLÓGICA CONVENCIONAL E AUTOMÁTICA
}

\author{
ANDRÉ SAMUEL STRASSBURGER, ANTÔNIO JOSÉ ELIAS AMORIM DE MENEZES, TÂNGELA \\ DENISE PERLEBERG, EBERSON DIEDRICH EICHOLZ, MARTA ELENA GONZALEZ MENDEZ, \\ EDGAR RICARDO SCHÖFFEL
}

\author{
Universidade Federal de Pelotas (UFPel), Faculdade de Agronomia Eliseu Maciel, Pelotas, RS, Brasil. \\ strassburger.as@gmail.com,menezes@cpatu.embrapa.br, tangelaperleberg@gmail.com, eeicholz@gmail. \\ com,marta@ufpel.tche.br, ricardo_schoffel@ufpel.edu.br
}

Recebido Janeiro 2009 - Aceito Outubro 2010

\begin{abstract}
RESUMO
Este trabalho teve como objetivos comparar os dados da temperatura do ar (temperatura média e extrema), obtidos em uma Estação Meteorológica Convencional com os de uma Estação Meteorológica Automática, e avaliar as estimativas da temperatura média diária do ar por diferentes métodos. As estações meteorológicas estavam localizadas na Estação Agroclimatológica de Pelotas, Capão do Leão (31 ${ }^{\circ} 52^{\prime}$ S, 52 $21^{\prime}$ 'W e 13,2 m), Rio Grande do Sul, Brasil. A série de cada elemento foi obtida simultaneamente na Estação Automática e na Estação Convencional durante o período de 22/12/2005 a 21/12/2006. Existe alta equivalência para as medidas das temperaturas extremas e das estimativas das temperaturas médias entre as Estações Meteorológicas Convencional e Automática, independente do método utilizado para o cálculo da temperatura média do ar. O termohigrógrafo mede, com alta precisão, as temperaturas extremas do ar. Verificam-se pequenas diferenças nos horários de ocorrência das temperaturas mínimas e máximas entre as Estações Meteorológicas Convencional e Automática. A análise dos dados indica que a substituição da Estação Meteorológica Convencional pela Estação Meteorológicas Automática, na região Sul do Rio Grande do Sul, não acarreta mudanças significativas na série de dados de temperatura do ar.
\end{abstract}

Palavras-chave: sensores de temperatura, temperatura média do ar, temperaturas extremas, termohigrógrafo.

\begin{abstract}
COMPARISON OF THE AIR TEMPERATURE OBTAINED BY CONVENTIONAL AND AUTOMATIC METEOROLOGICAL STATIONS

The objective of this work was to compare the air temperature data (average and extreme) obtained by a Conventional Meteorological Station and an Automatic Meteorological Station and evaluate different methods of estimating the average air temperature. The Meteorological Stations were located at the Agroclimatological Station of Pelotas, Capão do Leão (31 ${ }^{\circ} 52^{\prime}$ S, $52^{\circ} 21^{\prime}$ W and 13,2 m), Rio Grande do Sul, Brazil. The data series were collected simultaneously in Conventional Meteorological Station and Automatic Meteorological Station during the period from 12/22/2005 to $12 / 21 / 2006$. There is high equivalence in the measures of extreme temperature and average air temperature obtained from the Conventional and Automatic Meteorological Station, independently of the methods used for the calculation of average air temperature. The termo-hygrograph measures with high precision the extreme air temperature. There are small differences in the times of occurrence of the minimum and maximum temperatures between Conventional and Automatic Meteorological Stations. The data analysis indicated that the substitution of the Conventional Meteorological Station by Automatic Meteorological Station in Southern of Rio Grande do Sul does not changes significantly the air temperature data series. Keywords: temperature sensors, average air temperature, extreme temperature, termo-hygrograph.
\end{abstract}




\section{INTRODUÇÃO}

A temperatura do ar é um elemento ambiental de grande importância agrícola, pois exerce influência sobre diversos processos vitais das plantas, tais como fotossíntese, respiração e transpiração, portanto no crescimento vegetal e, sobre os estádios de desenvolvimento das culturas (Lucchesi, 1987), existindo faixas limite (temperaturas cardinais) para cada cultura, determinando a possibilidade ou sazonalidade da produção. A amplitude térmica é fator determinante para a produção agrícola, pois influencia na definição das épocas de semeadura, na escolha de cultivares e na adoção de práticas de manejo que busquem modificar o ambiente de cultivo.

As temperaturas máximas e mínimas estão associadas a outras variáveis meteorológicas, como: disponibilidade de energia solar, nebulosidade, umidade do ar e do solo, vento (direção e velocidade) e a parâmetros geográficos como topografia, altitude e latitude do local, além da cobertura e tipo de solo (Ometto, 1981; Pereira et al., 2002).

Vários autores citam que elevadas temperaturas do ar interferem na absorção de $\mathrm{CO}_{2}$, na distribuição de fotoassimilados, na produção de biomassa e no mecanismo de abertura e fechamento dos estômatos (Pereira et al., 1997; Andriolo, 1999; Taiz e Zeiger, 2004). As baixas temperaturas do ar são importantes em estudos sobre a ocorrência de geadas, considerando-se $2^{\circ} \mathrm{C}$ como o limite abaixo do qual iniciam os prejuízos a diversas culturas (Camargo et al., 1993). Diante da importância do conhecimento da dinâmica da temperatura do ar, dos valores e horários de ocorrência das mínimas e máximas, existe a necessidade de mensurar e registrar tais extremos. Além disso, muitos métodos de estimativa da evapotranspiração de cultivos agrícolas são obtidos em função da temperatura do ar, portanto a qualidade dessa informação poderá acarretar em erros na estimativa do consumo de água, conforme demonstraram Oliveira e Volpe (2003).

Devido ao avanço da tecnologia, principalmente na automação de dados meteorológicos, os instrumentos mecânicos existentes nas estações convencionais estão sendo substituídos por sensores automáticos. Os motivos vão desde a maior capacidade de amostragem até a possibilidade de operação em locais inóspitos e/ou de difícil acesso.

Entretanto, apesar das estações automáticas fornecerem dados com melhor caracterização das condições meteorológicas, em função da ação do tempo de uso, estão sujeitas a danos físicos (interferência no sinal, desconexão e oxidação de cabos, dentre outros) e, por consequência, nessas condições, poderão gerar dados imprecisos ou interrupção na série de dados. Assim, devido à diferença quanto ao número de amostragens entre as duas estações, a substituição de uma Estação Meteorológica Convencional por uma automatizada requer a homogeneização da série de dados.
O processo de substituição exige cuidado, pois existe a necessidade de homogeneizar a nova série com a antiga, ou seja, transferir confiabilidade de uma para a outra e, portanto, torná-la uma série única (Sentelhas et al., 1997; Seibert e Morén, 1999). Para Almeida et al. (2007), a substituição de uma Estação Meteorológica Convencional por uma Estação Meteorológica Automática não é um ato simples de troca, e por isso, requer obrigatoriamente os funcionamentos simultâneos e sua comparação. Este aspecto é ainda mais importante quando se considera a temperatura média em virtude das diferentes capacidades de amostragem das duas estações meteorológicas.

Estudos realizados por Fisch e Santos (1997), Sentelhas et al. (1997), Souza et al. (2003), Almeida et al. (2008) e Pereira et al. (2008) mostram que existe boa concordância entre os elementos meteorológicos obtidos nos sistemas automático e convencional, especialmente, a temperatura do ar. Na região de Botucatu, SP, Cunha e Martins (2004) encontraram alta concordância entre os dados de temperaturas do ar obtidas na Estação Meteorológica Convencional em relação aos da Estação Meteorológica Automática, mas recomendam que a substituição de um sistema por outro só deva ser realizada após análise comparativa detalhada entre as duas estações. Embora esses trabalhos demonstrem alta concordância entre os dados obtidos pelas duas estações climatológicas, os coeficientes de ajuste devem ser calculados de forma independente para cada local em que as Estações Climatológicas estejam instaladas.

Considerando que as condições climáticas do Sul do Rio Grande do Sul apresentam consideráveis diferenças em relação ao clima das demais regiões do Brasil, este trabalho teve como objetivos comparar os dados da temperatura do ar (temperatura média e extrema) obtidos em uma Estação Meteorológica Convencional com os de uma Estação Meteorológica Automática, e avaliar as estimativas da temperatura média diária do ar por diferentes métodos em Pelotas, Rio Grande do Sul Brasil.

\section{MATERIAL E MÉTODOS}

Os dados da temperatura do ar foram obtidos na Estação Agroclimatológica de Pelotas localizada no município de Capão do Leão, RS, Brasil ( $31^{\circ} 52^{\prime} \mathrm{S}, 52^{\circ} 21^{\prime} \mathrm{W}$ e $13,2 \mathrm{~m}$ de altitude). As séries avaliadas correspondem a observações realizadas na Estação Meteorológica Convencional (EMC) e na Estação Meteorológica Automática (EMA), durante o mesmo período, de 22 de dezembro de 2005 a 21 de dezembro de 2006.

Os sensores de temperatura do ar da EMC e da EMA estavam instalados na mesma área de estudo. Os abrigos termométricos nos quais estavam instalados os sensores de temperatura do ar de ambas as estações estavam de acordo com as normas estabelecidas pela Organização Mundial de 
Meteorologia (OMM; WMO, 1983). A EMA estava programada para medir a temperatura do ar a cada segundo e armazenar a média a cada 30 minutos. Os equipamentos utilizados nas duas estações, elementos sensor, e suas sensibilidades, precisão e o método de cálculo das médias são apresentados na Tabela 1 .

Os dados diários corresponderam ao período entre às 0 TMG (Tempo Médio de Greenwich; $21 \mathrm{~h}$ local do dia $n-1)$ e às $24 \mathrm{TMG}(21 \mathrm{~h}$ do dia $n)$, conforme metodologia adotada pelo Instituto Nacional de Meteorologia (INMET) e as recomendações da WMO (1983).

Compararam-se os dados diários obtidos na EMC com os dados obtidos na EMA referentes à temperatura máxima, à temperatura mínima e à temperatura média. Em relação à temperatura média diária, foram realizadas três comparações: i) temperatura média diária real, considerando-se a média das 86.400 leituras da EMA, comparada à temperatura média da EMC calculada pela equação padrão da WMO e, utilizada pelo INMET; ii) média calculada pela equação padrão da WMO, em ambas as estações e; iii) média calculada pelas temperaturas extremas, em ambas as estações. Ainda, foi determinada a relação entre os dados de temperaturas extremas do ar (máxima e mínima) obtidas por termohigrógrafo com os respectivos dados medidos por termômetros na EMC e na EMA.

Para comparar os dados meteorológicos obtidos nos dois sistemas foram realizadas análises de tendência central e de dispersão. As equações de regressão linear simples entre os dados de temperatura média, máxima e mínima foram escolhidas com base no indicativo de precisão e maior coeficiente de determinação utilizando-se planilhas eletrônicas. Os resultados obtidos da análise de regressão foram avaliados por meio dos coeficientes linear (a), angular (b) e de determinação $\left(r^{2}\right)$, que traduzem exatidão máxima quando $\mathrm{a}=0 \mathrm{e} \mathrm{b}=1$ e precisão máxima quando $r^{2}=1$.

\section{RESULTADOS E DISCUSSÃO}

As regressões lineares simples e os respectivos coeficientes de determinação $\left(\mathrm{r}^{2}\right)$ entre os dados obtidos pela EMC e a EMA para as variáveis temperatura máxima, temperatura mínima (Figura 1) e temperatura média (Figura 2) mostraram a existência de elevada precisão ( $\mathrm{r}^{2}$ acima de $\left.95 \%\right)$ entre os valores obtidos nas duas estações meteorológicas.

Conforme pode ser observado na Figura 1a-b, as medições obtidas por EMA e EMC apresentaram alta correlação para os dados de temperatura máxima e mínima do ar, sendo que as equações ajustadas proporcionaram coeficientes de alta precisão $\left(\mathrm{r}^{2}=0,99\right)$ e exatidão $(\mathrm{a}=0 \mathrm{e} \mathrm{b}$ próximo $\mathrm{a} 1)$, indicando que para essas variáveis pode-se utilizar os dados tanto da EMC como da EMA. Esses resultados corroboram aqueles obtidos por Pereira et al. (2008), que obtiveram alta correlação das medidas entre sensores automáticos e convencionais com $\mathrm{r}^{2}=0,9925$, $\mathrm{a}=0,1962$ e $\mathrm{b}=0,9966$, para a temperatura máxima; e de $\mathrm{r}^{2}=$ $0,9947, a=-0,113$ e $b=1,0074)$.

Uma das metodologias mais utilizadas para medir as temperaturas máximas e mínimas do ar é a utilização do termógrafo ou ainda do termohigrógrafo. Embora esses equipamentos apresentem menor precisão, tem como principal vantagem o menor custo em relação aos equipamentos necessários para realizar as leituras extremas de uma EMC e de uma EMA e, ainda, com relação à EMC, tem a vantagem de registrar continuamente as flutuações da temperatura do ar. Sua menor precisão deve-se, principalmente, por erros no manuseio e na menor sensibilidade do elemento sensor. Entretanto, esse equipamento é de fácil aquisição, manuseio e instalação. Mesmo assim, as comparações das temperaturas extremas obtidas por termohigrógrafo na EMC, em relação àqueles medidos por termômetro na EMC e na EMA, representadas na Figura 1c-f,

Tabela 1 - Descrição dos sensores das estações meteorológicas automática (EMA) e convencional (EMC), fabricante, sensibilidade/precisão e métodos de cálculo das médias dos dados obtidos. Pelotas, 2010.

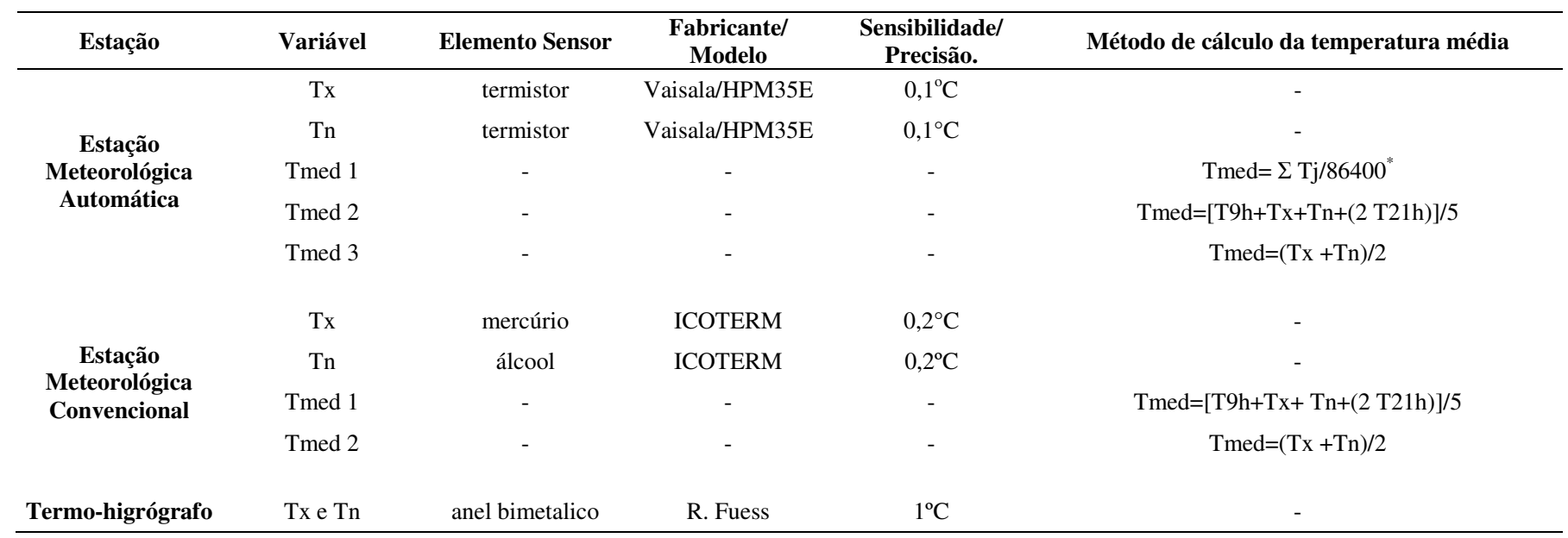


possuem alto coeficiente de determinação (acima de 95\%), indicando alta precisão e com boa exatidão $(\mathrm{a}=0$ e b próximo de 1). Dessa forma, os termohigrógrafos podem ser utilizados, com certa exatidão e precisão, para a estimativa das temperaturas médias calculadas pela equação das extremas.

Em relação às temperaturas médias, observou-se que existe maior dispersão dos dados, com discreta redução no coeficiente de determinação da equação, quando estes são calculados a partir da medição a cada segundo na EMA e comparados aos valores da EMC calculados pela equação padrão do WMO (Figura 2a). Essa diferença pode ser atribuída à metodologia adotada para o cálculo da temperatura média, uma vez que para a EMA o valor deriva de 86.400 leituras coletadas ao longo do dia, enquanto que na EMC esse valor resultou de quatro leituras diárias. Quando a metodologia de cálculo da temperatura foi a mesma (pela equação padrão da WMO e pela equação das extremas) a diferença de valores foi menor entre a EMC e EMA, conforme pode ser visualizado pela dispersão de dados da Figura 2b-c e pelos altos coeficientes das equações de ajuste. Com isso, é possível deduzir que os procedimentos, adotados no cálculo da temperatura média, podem gerar maiores diferenças no valor real do que os sensores utilizados para a medida. Pereira et al. (2008) comparando dados de EMC e EMA em Londrina, encontraram maior dispersão de dados de temperatura média, do que para as temperaturas máximas e mínimas.
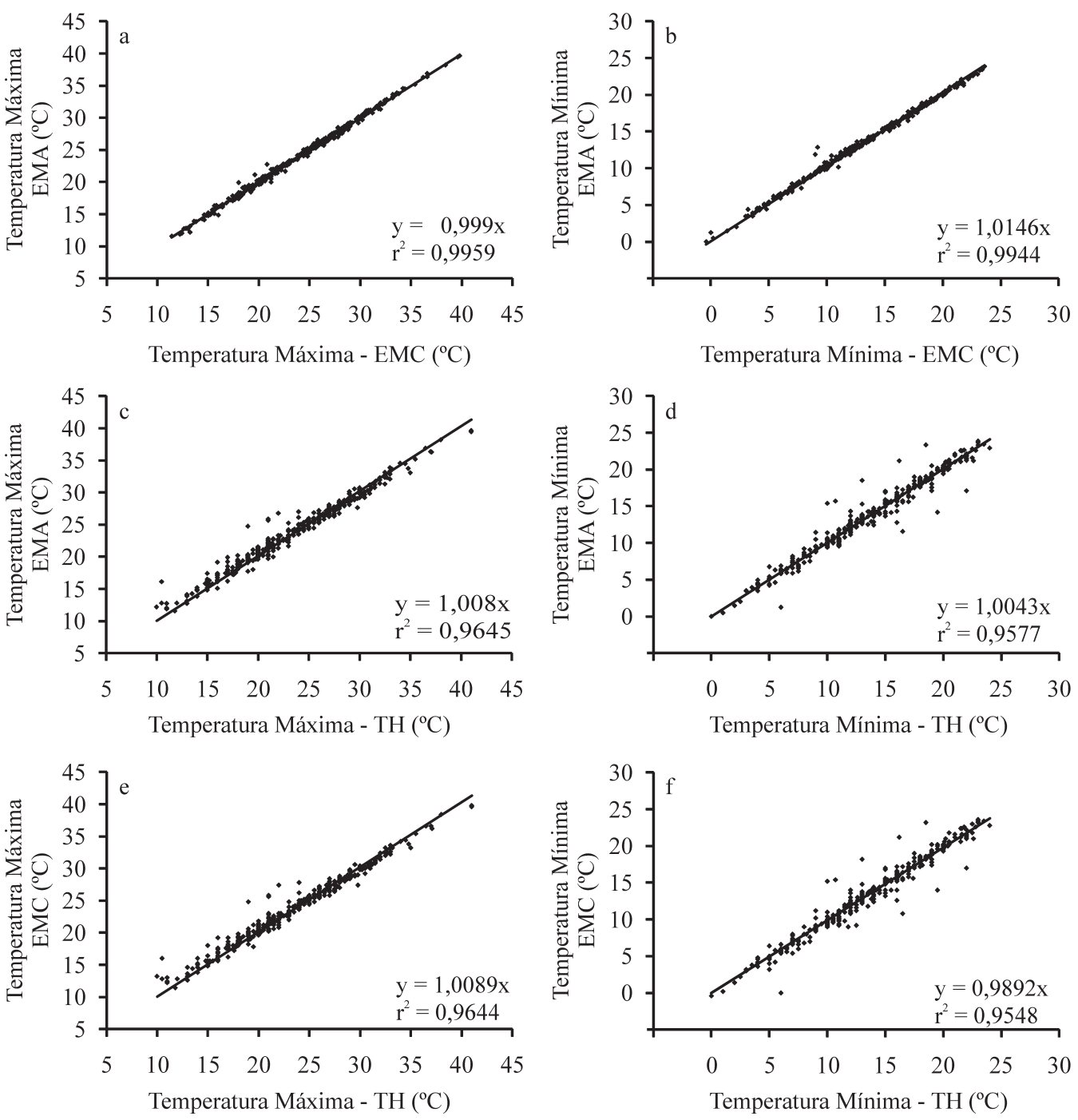

Figura 1- Relações das temperaturas máximas (a; c; e) e das temperaturas mínimas (b; d; f) do ar entre a Estação Meteorológica Convencional (EMC) e Automática (EMA - a; b), entre a Estação Meteorológica Automática e o Termohigrógrafo (TH - c; d) e entre a Estação Meteorológica Convencional e o Termohigrógrafo (e; f), no período de 22/12/05 e 21/12/06. Pelotas, 2010. 

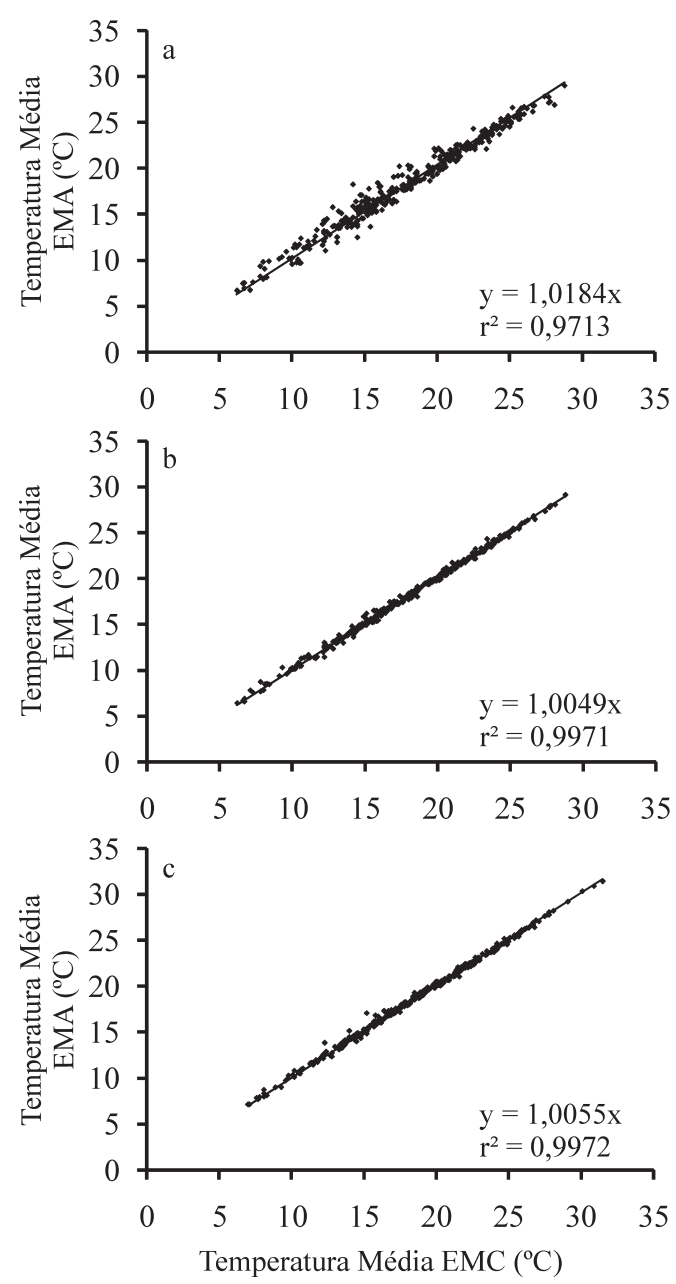

Figura 2- Relação entre as temperaturas médias mensuradas por Estação Meteorológica Convencional (EMC) e por Estação Meteorológica Automática (EMA), no período de 22/12/05 e 21/12/06. (a) Temperaturas média da EMA calculada a partir de 86.400 dados diários e temperaturas média da EMC calculada pela equação padrão do WMO; (b) Temperaturas média da EMA e da EMC calculadas pela equação padrão do WMO e; (c) Temperaturas médias obtidas pela equação das temperaturas extremas. Pelotas, 2010.

Quanto aos horários de ocorrência da temperatura máxima e mínima (Figura 3), observou-se que para ambas as estações meteorológicas houve concentração de ocorrências em alguns horários do dia. Percebeu-se, porém, que ocorrem discrepâncias no número de ocorrências entre EMC e a EMA em determinados horários do dia.

Para a temperatura máxima o período de ocorrência foi entre as 12:00 e 16:30 h em ambas as estações meteorológicas. Observou-se que o maior número de ocorrência das máximas na EMC foi por volta das 15:00 h, mas isto não é tão nítido na EMA. Para a temperatura mínima o período de ocorrência fica entre as 19:30 h do dia $\mathrm{n}$ e 9:00 h do dia $n+1$, ocorrendo maior frequência nos horários próximos às $6: 00 \mathrm{~h}$, ou seja, próximo ao amanhecer.
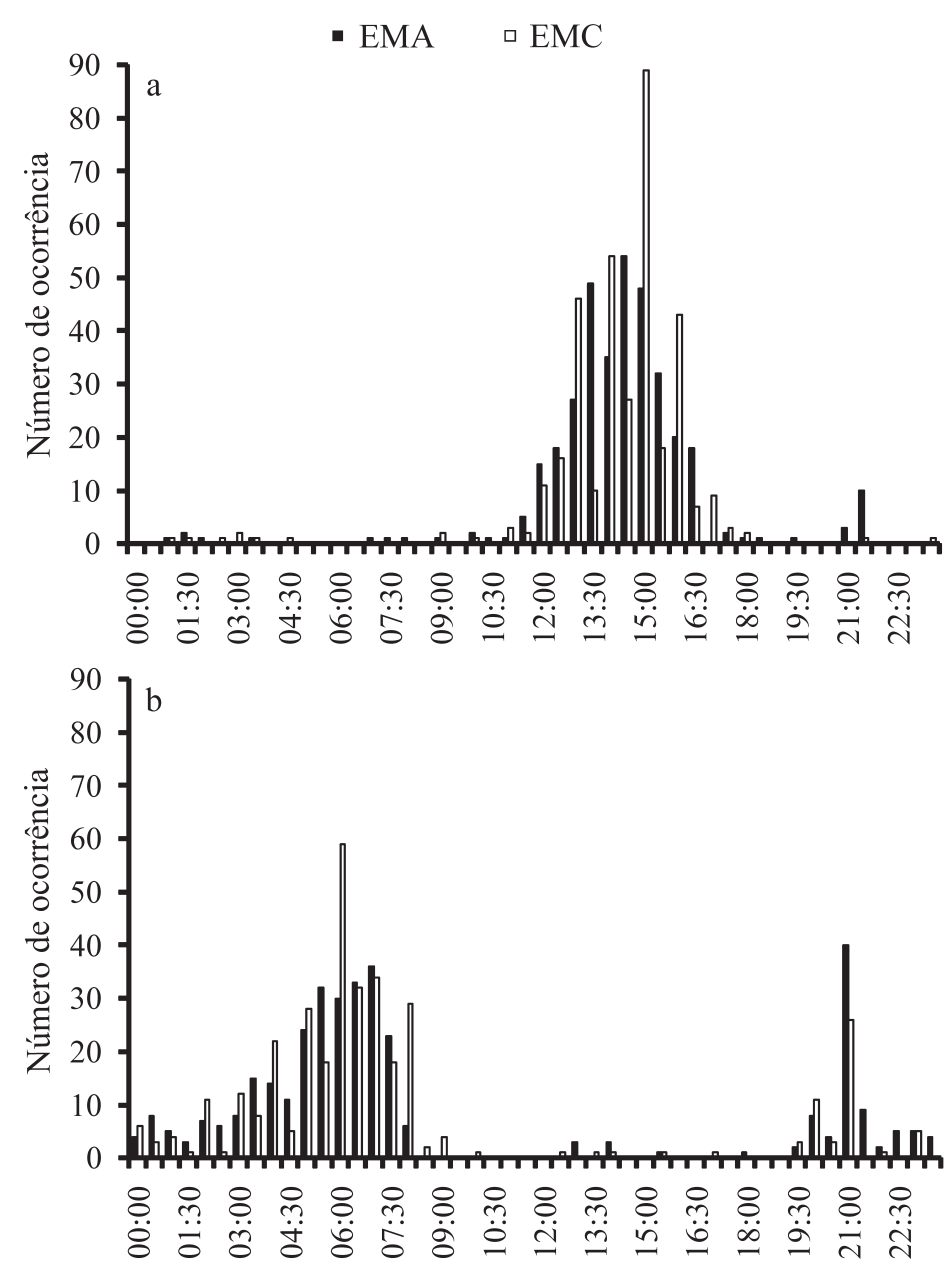

Horário

Figura 3- Horário de ocorrência da temperatura máxima (a) e da temperatura mínima (b) obtidas na Estação Meteorológica Automática (EMA) e na Estação Meteorológica Convencional (EMC) durante o período de 22/12/05 e 21/12/06. Pelotas, 2010.

A diferença entre os horários de ocorrência das extremas na EMC e na EMA podem ser atribuídas à diferença de sensibilidade entre os sensores e, ainda, à metodologia utilizada para analisar os dados na EMC. Nessa, os horários são lidos a partir de picos de temperatura em gráficos registrados por termohigrógrafo, os quais muitas vezes não são suficientemente nítidos e necessitam de perfeito ajustamento do papel ao tambor rotativo, além de que, esse diagrama possui escala reduzida de resolução de temperatura $\left({ }^{\circ} \mathrm{C}\right)$ e de tempo (horas), as quais acabam contribuindo para que ocorram erros no registro e na interpretação (leitura) dos valores desse equipamento.

Na Figura 4 são apresentadas as médias mensais da temperatura máxima, mínima e médias das estações automática e convencional. A análise comparativa dos dados revela que as diferenças entre essas variáveis foram pequenas, sendo que a maior diferença entre as estações meteorológicas foi de $0,5^{\circ} \mathrm{C}$, observada para a temperatura média das mínimas no mês 


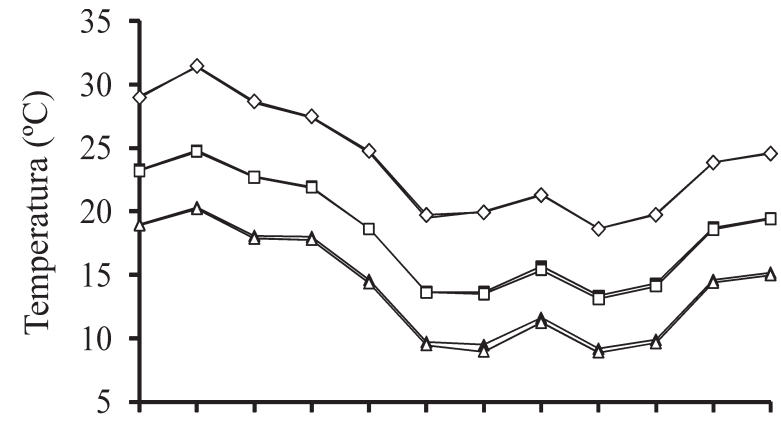

Dez Jan Fev Mar Abr Mai Jun Jul Ago Set OutNov

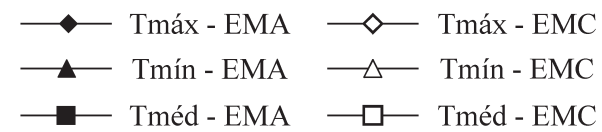

Figura 4 - Valores médios mensais das temperaturas máxima, mínima, média (calculada pela equação padrão do WMO) obtidas na Estação Meteorológica Automática (EMA) e na Estação Meteorológica Convencional (EMC) durante o período de 22/12/05 e 21/12/06. Pelotas, 2010.

de junho. Quanto aos dados médios de temperatura mínima, percebem-se valores ligeiramente superiores na EMA em relação aos da EMC, principalmente nos meses de inverno (junho a setembro). Estes resultados diferem daqueles encontrados por Sentelhas et al. (1997) e Fisch e Santos (1997), na região Sudeste do Brasil, que observaram tendência dos dados obtidos pela EMC serem mais elevados do que os dados obtidos na EMA.

\section{CONCLUSÃO}

- Existe alta equivalência entre as medidas das temperaturas extremas das Estações Meteorológicas Convencional e Automática;

- Existe alta concordância entre as estimativas da temperatura médias diária do ar nas Estações Meteorológicas Convencional e Automática independente do método utilizado;

- O termohigrógrafo mede, com alta precisão, as temperaturas extremas do ar;

- Verificam-se pequenas diferença nos horários de ocorrência das temperaturas mínimas e máximas entre as Estações Meteorológicas Convencional e Automática;

- A análise dos dados indica que a substituição da Estação Meteorológica Convencional pela Estação Meteorológicas Automática, na região Sul do Rio Grande do Sul, não acarreta mudanças significativas na série de dados de temperatura do ar.

\section{REFERÊNCIAS BIBLIOGRÁFICAS}

ALMEIDA, H.A. de; SOUZA, J.A. de; ALCÂNTRA, H.M. de. Análise comparativa de dados de temperatura do ar obtidos por estações meteorológicas convencional e automática. In: CONGRESSO
BRASILEIRO DE AGROMETEOROLOGIA, 15, 2007, Aracaju. Anais... 2007. CD-ROM.

ALMEIDA, H.A.; SOUZA, J.A.; ALCÂNTARA, H.M. Análise comparativa de dados meteorológicos obtidos por estação convencional e automática no semi-árido paraibano. Revista Brasileira de Agrometeorologia, v. 16, n. 1, p. 58-66, 2008.

ANDRIOLO, J.L. Fisiologia das culturas protegidas. Santa Maria: UFSM, 1999. 142p.

CAMARGO, M.B.P.; PEDRO JUNIOR, M.J.; ALFONSI, R.R. Probabilidade de ocorrências de temperaturas mínimas absolutas mensais e anuais no Estado de São Paulo. Bragantia, v. 52, n. 2, p. 161-68, 1993.

CUNHA, A.R., MARTINS, D. Estudo comparativo entre elementos meteorológicos obtidos em estações meteorológicas convencional e automática em Botucatu, SP, Brasil. Revista Brasileira de Agrometeorologia, v. 12, n. 1, p. 103-111, 2004.

FISCH, G., SANTOS, J.M. Comparação entre observações meteorológicas convencionais e automáticas na região do Vale do Paraíba, SP. In: CONGRESSO BRASILEIRO DE AGROMETEOROLOGIA, 10. 1997, Piracicaba. Anais... p. 246-248, 1997.

LUCCHESI, A.A. Fatores da produção vegetal. In: CASTRO, P.R. Ecofisiologia da produção agrícola. Piracicaba: Associação Brasileira para Pesquisa da Potassa e do Fosfato, 1987. p. 1-11.

OLIVEIRA, A.D.; VOLPE, C.A. Comparação de métodos de estimativa da evapotranspiração de referência, utilizando dados de estações meteorológicas convencional e automática. Revista Brasileira de Agrometeorologia, v. 11, n. 2, p. 253-260, 2003.

OMETTO, J.C. Bioclimatologia vegetal, São Paulo: Ceres, 1981. 440p. PEREIRA, A.R.; ANGELOCCI, L.R.; SENTELHAS, P.C. Agrometeorologia: fundamentos e aplicações práticas. Guaíba: Agropecuária, 2002. 478p.

PEREIRA, A.R.; VILLA NOVA, N.A.; SEDIYAMA, G.C. Evapo(transpi)ração. Piracicaba: FEALQ, 1997. 183p.

PEREIRA, L.M.P.; CARAMORI, P.H.; RICCE, W.S.; CAVIGLIONE, J.H. Análise comparativa de dados meteorológicos obtidos por estação convencional e automática em Londrina. Semina: Ciências Agrárias, v. 29, n. 2, p. 299-306, 2008.

SEIBERT, J.; MORÉN, A.S. Reducings systematic errors in rainfall measurements using a newtype of gauge. Agricultural and Forest Meteorology, v.98-99, n. 1, p. 341-348, 1999.

SENTELHAS, P.C.; MORAES, S.O.; PIEDADE, S.M.S.; PEREIRA, A.R.; ANGELOCCI, L. R.; MARIN, F.R. Análise comparativa de dados meteorológicos obtidos por estações convencional e automática. Revista Brasileira de Agrometeorologia, v. 5, n. 2, p. 215-221, 1997.

SOUZA, I.A.; GALVANI, E.; ASSUNÇÃO, H.F. Estudo comparativo entre elementos meteorológicos monitorados por estações convencional e automática na Região de Maringá. Acta Scientiarum. Technology, v. 25, n. 2, p. 203-207, 2003.

TAIZ, L.; ZEIGER, E. Plant physiology. $3^{\mathrm{a} e d}$. Sunderland: Sinauer Associates, 2004.

WMO. Guide to meteorological instruments and methods of observation. Geneva, World Meteorological Organization, n. 8, $5^{\mathrm{a}}$ ed., 1983. 230p. 